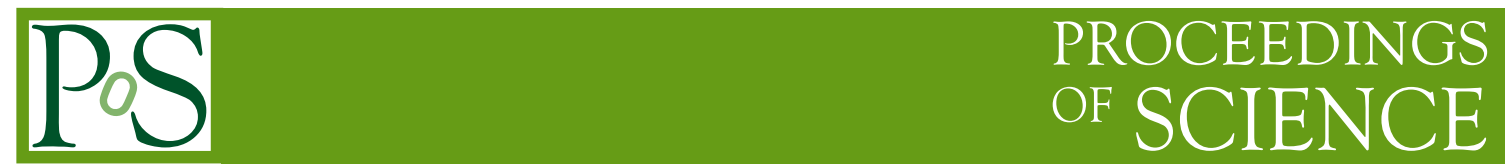

\title{
4th generation searches at ATLAS
}

\section{Lorenzo Feligioni*}

On behalf of the ATLAS Collaboration

CNRS IN2P3 AMU

E-mail: lorenzo@cppm.in2p3.fr

We present searches for fourth generation quarks in data corresponding to an integrated luminosity of $1.04 \mathrm{fb}^{-1}$ from $p p$ collisions delivered by the LHC at $\sqrt{s}=7 \mathrm{TeV}$ recorded with the ATLAS detector. With three different analyses presented in this paper, ATLAS excludes at the $95 \%$ confidence level a heavy down-type quark, heavy up-type quark and a more general heavy-quark with mass less than $480 \mathrm{GeV}, 404 \mathrm{GeV}$ and $350 \mathrm{GeV}$, respectively.

36th International Conference on High Energy Physics

4-11 July 2012

Melbourne, Australia

* Speaker. 


\section{Introduction}

One of the most natural extensions to the Standard Model is represented by increasing by one or more the heavy quark sector. This is still compatible with Electroweak precision measurements [1], assuming a small mass splitting between the heavy up-type quark $\left(t^{\prime}\right)$ and the heavy down-type quark $\left(b^{\prime}\right)$, and would provide an additional source of CP violation needed to explain the baryon asymmetry of the universe. Extra heavy quarks can be pair produced and searched for in the products of high energy collisions taking place at hadron colliders. Previous searches have been carried out by the $\mathrm{D} \emptyset$ and CDF experiments at Fermilab [2, 3, 4 .

In this paper we present three complementary searches for fourth-generation quarks performed with the ATLAS detector using $1.04 \mathrm{fb}^{-1}$ of 2011 data. The channels considered are:

- $t^{\prime} \bar{t}^{\prime} \rightarrow W^{+} W^{-} b \bar{b}$ in the lepton plus jets channel [5]; assuming that $m_{t^{\prime}}-m_{b^{\prime}}<m_{W}$, where $m_{W}$ is the $W$ boson mass, the $t^{\prime}$ quark predominantly decays into a $W$ boson and a down-type quark $q(q=d, s, b)$;

- $b^{\prime} \bar{b}^{\prime} \rightarrow W^{+} W^{-} t \bar{t} \rightarrow W^{+} W^{-} W^{+} W^{-} b \bar{b}$ in the lepton plus jets channel [6]; indeed if $b^{\prime}$ is chiral and its mass is larger than $m_{t}+m_{W}$, then it decays predominantly as $b^{\prime} \rightarrow W t \rightarrow W W b$;

- a general heavy quark $(Q \bar{Q})$ pair production, decaying via $Q \rightarrow W q$ where $q=d, s, b$ for up-type $Q$ or $q=u, c$ for down-type $Q$, in the dilepton final state [7].

\section{Event Selection}

The dataset used in this analysis was recorded between March and June 2011 with the ATLAS detector [8] using single electron and muon triggers. The corresponding integrated luminosity is $1.04 \mathrm{fb}^{-1}$. Electron candidates are required to have a transverse momentum cut of $p_{\mathrm{T}}>25 \mathrm{GeV}$ and pseudorapidity, $|\eta|<2.47$, excluding the transition region $1.37<|\eta|<1.52$ between the barrel and endcap electromagnetic calorimeters. Muon candidates are required to satisfy $p_{\mathrm{T}}>20 \mathrm{GeV}$ and $|\eta|<2.5$. For the lepton plus jets analyses the background from multi-jet production is suppressed by requiring a missing transverse energy $E_{\mathrm{T}}^{\text {miss }}>35(20) \mathrm{GeV}$ in the electron (muon) channel, followed by $E_{\mathrm{T}}^{\text {miss }}+m_{\mathrm{T}}>60 \mathrm{GeV}$, where $m_{\mathrm{T}}$ is the transverse mass of the lepton and $E_{\mathrm{T}}^{\text {miss }}$. In the dilepton analysis same-flavor events $\left(e e\right.$ and $\mu \mu$ ) must satisfy $E_{\mathrm{T}}^{\text {miss }}>60 \mathrm{GeV}$ to suppress backgrounds from $Z / \gamma^{*} \rightarrow \ell \ell$ decays. For the same reason the dilepton invariant mass of sameflavor events (ee and $\mu \mu$ ) must be greater than $15 \mathrm{GeV}$ and must fall outside a window around the $Z$ boson mass, defined as $81 \mathrm{GeV}<m_{\ell \ell}<101 \mathrm{GeV}$. Also for the dilepton analysis differentflavor events $(e \mu), H_{\mathrm{T}}$, defined as the scalar sum of $E_{\mathrm{T}}$ from every lepton and jet passing the object selection criteria, must exceed $130 \mathrm{GeV}$. The $H_{\mathrm{T}}$ requirement reduces the $Z / \gamma^{*} \rightarrow \tau \tau$ background, where the $E_{\mathrm{T}}^{\mathrm{miss}}$ requirement is insufficient due to the presence of neutrinos.

Further jet requirements are different for each of the three analyses: the $t^{\prime}$ analysis demands at least three jets with $p_{\mathrm{T}}>25 \mathrm{GeV}$ and $|\eta|<2.5$, with at least one jet satisfying $p_{\mathrm{T}}>60 \mathrm{GeV}$. The requirement for the dilepton $Q \bar{Q}$ analysis asks for at least two jets, while the $b^{\prime}$ analysis demands at least six jets. Finally, to further reduce the backgrounds, the $t^{\prime}$ analysis requires at least one jet to be identified as originating from the hadronization of a $b$ quark ( $b$-tagging). 


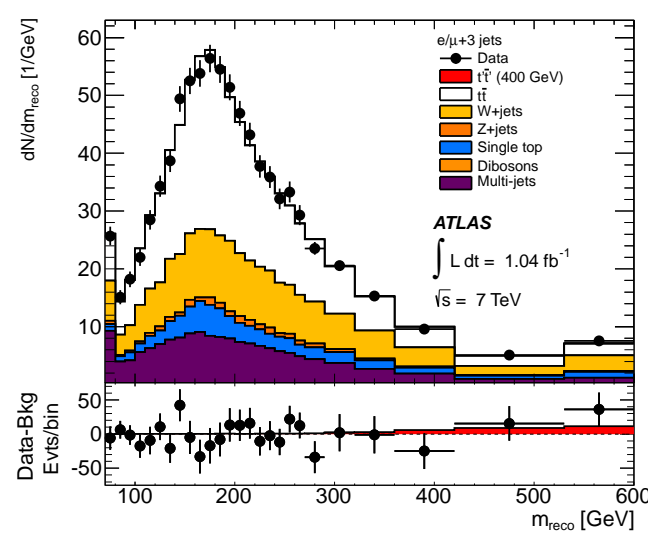

(a)

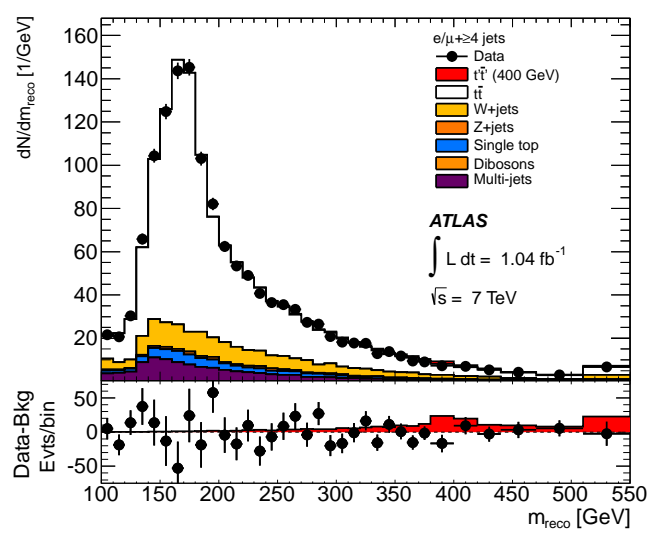

(b)

Figure 1: The reconstructed mass $m_{\text {reco }}$ distribution in the combined (a) $e / \mu+3$ jets and (b) $e / \mu+\geq 4$ jets channels. The data (points) are compared to the SM background predictions using the values of the nuisance parameters obtained from the fit to data under the background-only hypothesis (stacked histograms). In the top panels the bin contents have been divided by bin width. The bottom panels show the backgroundsubtracted data distribution. The underflow and overflow have been folded into the first and last bins, respectively. Also shown is the expected contribution from a signal with mass $m_{t^{\prime}}=400 \mathrm{GeV}$ (histogram) [5].

\section{Signal and background discrimination}

In the $t^{\prime}$ analysis the main background after the event selection consists of $t \bar{t}$ production, followed by the production of a $W$ boson in association with jets $(W+$ jets), multi-jet events, single top quark, $Z+$ jets and diboson production. The reconstructed top mass $\left(m_{\text {reco }}\right)$ is the primary discriminating variable: for events with $\geq 4$ jets, $m_{\text {reco }}$ is estimated by performing a kinematic likelihood fit to the $t^{\prime} \bar{t}^{\prime} \rightarrow W^{+} b W^{-} \bar{b} \rightarrow \ell v b q \bar{q}^{\prime} \bar{b}$ hypothesis, imposing the constraints that $t^{\prime}$ and $\bar{t}^{\prime}$ have the same mass, and that the mass of the lepton-neutrino system, as well as that of a jet pair, equals the nominal $W$ boson mass. Among all possible jet-parton permutations, the one yielding the highest likelihood value after maximization over the fit parameters is kept. In the case of events with exactly three jets, $m_{\text {reco }}$ is taken to be the invariant mass of the three-jet system.

After the first lepton plus jets selection, pair production of $b^{\prime}$ quarks is distinguished mostly by the large number of energetic jets. Events with $b^{\prime}$ decays contain jets from three hadronic $W$ decays, while $t \bar{t}$ background events contain only one hadronic $W$ decay. To identify these hadronic $W$ decays, pairs of jets separated by $\Delta R<1.0$ are examined. This choice of $\Delta R$ selects $W$ bosons with high $p_{\mathrm{T}}$ and reduces the combinatorial background in events with large jet multiplicity. The number of reconstructed $W$ bosons $\left(N_{W}\right)$ is defined as the number of such jet pairs with an invariant mass in the range $70-100 \mathrm{GeV}$. Each jet may contribute to only one identified hadronic $W$ decay.

Finally in the heavy quark $Q \bar{Q}$ analysis, after the baseline selection described in Section 2, the mass reconstruction of heavy quark candidates is performed in order to discriminate the heavyquark decays from the dominant $t \bar{t}$ background. Direct reconstruction is not possible, as two neutrinos escape the detector. However, both neutrino momentum vectors are reconstructed by assuming that the neutrinos are the sole contributors to $E_{\mathrm{T}}^{\text {miss }}$ and that they are approximately collinear with the leptons. The optimal values of each $|\Delta \eta(v, \ell)|$ and each $|\Delta \phi(v, \ell)|$ are fit by minimizing 
the mass difference between the two reconstructed heavy quarks. The final reconstructed mass ( $\left.m_{\text {Collinear }}\right)$ is taken to be the average of the two reconstructed masses in the event. Even though this baseline selection provides excellent discrimination against $Z / \gamma^{*}$ production and other backgrounds, additional selection requirements are necessary to suppress the dominant $t \bar{t}$ background. A triangular selection in $H_{\mathrm{T}}+E_{\mathrm{T}}^{\text {miss }}$ versus $m_{\text {Collinear }}, H_{\mathrm{T}}+E_{\mathrm{T}}^{\text {miss }}>X-0.4 \times m_{\text {Collinear }}$ with $X$ dependent on the assumed signal mass, is applied. Mass-dependent requirements on $E_{\mathrm{T}}^{\mathrm{miss}}$ and leading jet $p_{\mathrm{T}}$ are imposed as well. These selection requirements are optimized in MC simulation by seeking a point of maximum significance, $S / \sqrt{S+B}$, while simultaneously varying all of the selection requirement parameters.

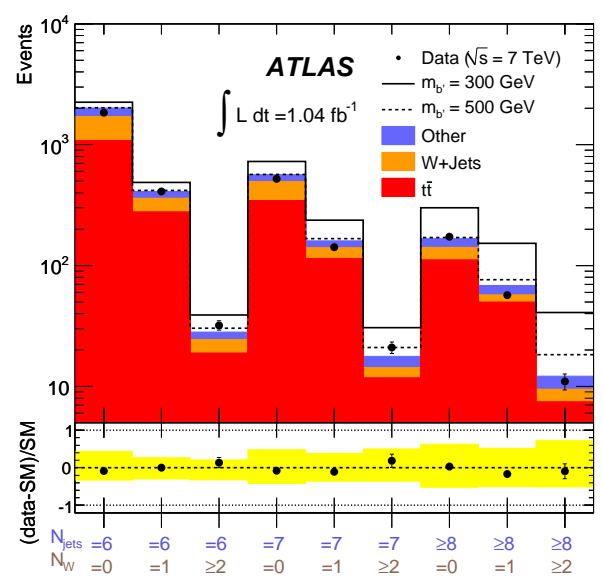

Figure 2: Distribution of the numbers of events observed in the data and expected from SM processes for jet multiplicity $N_{\text {jets }}=6,7, \geq 8$ with hadronic $W$ multiplicity $N_{W}=0,1, \geq 2$. The expected $b^{\prime}$ signals for two masses are also shown, stacked on top of the backgrounds [6].

\section{Results}

The reconstructed mass $m_{\text {reco }}$ distribution of $t^{\prime}$ candidates is analyzed using a log-likelihood ratio $L L R=-2 \log \left(L_{\mathrm{s}+\mathrm{b}} / L_{\mathrm{b}}\right)$ as test-statistic, where $L_{\mathrm{s}+\mathrm{b}}\left(L_{\mathrm{b}}\right)$ is a Poisson likelihood to observe the data under the signal-plus-background (background-only) hypothesis. The per-bin signal and background predictions are parameterized in terms of 12 nuisance parameters, describing the effect of leading sources of systematic uncertainty such as jet energy scale, initial (ISR) and final state radiation (FSR), and $t \bar{t}, W+$ jets and QCD multi-jet normalizations. The set of fitted nuisance parameters is chosen based on their overall impact on the search sensitivity, the expected constraining power of the data and their suitability to be treated as continuous parameters. Figure 1 shows a comparison of the post-fit $m_{\text {reco }}$ distribution between data and the background prediction for the combined $e / \mu+3$ jets and $e / \mu+\geq 4$ jets channels. In the absence of any significant data excess, neither in the $e+$ jets nor in the $\mu+$ jets channels individually, or in their combination, $95 \%$ confidence level (CL) upper limits on the $t^{\prime} \bar{t}^{\prime}$ production cross section are derived and translated on limits on the $t^{\prime}$ mass: at 95\% CL, a lower limit of 404 (394) GeV on the mass of the $t^{\prime}$ quark is then derived, whereas the expected $95 \%$ CL lower limit is $394 \mathrm{GeV}$. 
The expected background and signal contributions in the $b^{\prime}$ analysis are shown in Fig. for the nine bins of jet and hadronic $W$-boson multiplicity. The main contributions to the uncertainty in the modeling of the backgrounds and $b^{\prime}$ signal come from the jet energy scale and the level of ISR/FSR in the top quark pair background. The systematic uncertainties are treated as correlated between signal and background, and between electron and muon channels, except where they are specific to the background model (e.g. $W+$ jets normalization) or to a channel (e.g. electron or muon efficiencies). To extract the most likely value of the $\bar{b}^{\prime} b^{\prime}$ cross section in the nine bins of $\left(N_{W}, N_{\text {jet }}\right)$ multiplicity, a binned maximum likelihood fit using a profile likelihood ratio is performed, varying each background rate within its uncertainty, and allowing shape and rate variation due to the systematic uncertainties described above. The signal and background rates are fitted simultaneously. Since no signal is observed, for this analysis 95\% CL upper limits on the $\bar{b}^{\prime} b^{\prime}$ cross section exclude $b^{\prime}$ masses below $480 \mathrm{GeV}$ at the $95 \% \mathrm{CL}$, while the expected limit is $m_{b^{\prime}}>470 \mathrm{GeV}$.

Finally, a binned maximum-likelihood ratio technique is used to fit distributions of $m_{\text {Collinear }}$ to the observed data in order to measure the most likely $Q \bar{Q}$ production cross-section, $\sigma(p p \rightarrow Q \bar{Q})$. Again, in presence of no signal, the upper limit on the production cross-section is converted into a lower limit on $m_{Q}$ : a lower limit of $m_{Q}$ is found to be $350 \mathrm{GeV}$ at $95 \% \mathrm{CL}$ whereas a limit of $m_{Q}>335 \mathrm{GeV}$ was expected.

\section{Conclusions}

In summary, searches for fourth generation quarks have been performed using $1.04 \mathrm{fb}^{-1}$ from $p p$ collisions at $\sqrt{s}=7 \mathrm{TeV}$ recorded with the ATLAS detector. No significant excess of events was found in any of the analyses reported in this paper. Consequently, lower limits on fourth generation quark masses have been set. For the heavy up-type quark searches the analysis finds $m_{t^{\prime}}>404 \mathrm{GeV}$ at $95 \% \mathrm{CL}$. The heavy down-type quark is also exlcuded at 95\% CL for masses below $480 \mathrm{GeV}$. Finally, the more general heavy quark searches analysis finds a lower limit of $m_{Q}>350 \mathrm{GeV}$ at 95\% CL.

\section{References}

[1] See e.g. B. Holdom et al., PMC Physics A 3, 4 (2009) and references therein.

[2] The CDF Collaboration, Search for a Heavy Toplike Quark in $\bar{p} p$ Collisions at $\sqrt{s}=1.96$ TeV, Phys. Rev. Lett. 107, 261801 (2011).

[3] The CDF Collaboration, Search for Heavy Bottom-like Quarks Decaying to an Electron or Muon and Jets in $p$ anti-p Collisions $\sqrt{s}=1.96$ TeV Phys. Rev. Lett. 106, 141803 (2011).

[4] The D0 Collaboration, Search for a Fourth Generation $t^{\prime}$ Quark in p $\bar{p}$ Collisions at $\sqrt{s}=1.96 \mathrm{TeV}$, Phys. Rev. Lett. 107, 082001 (2011).

[5] ATLAS Collaboration, Search for Pair Production of a Heavy Quark Decaying to a W Boson and a $b$ Quark in the Lepton+Jets Channel with the ATLAS Detector, Phys.Rev.Lett. 108 (2012) 261802.

[6] ATLAS Collaboration, Search for down-type fourth generation quarks with the ATLAS Detector in events with one lepton and high transverse momentum hadronically decaying W bosons in $\sqrt{s}=7 \mathrm{TeV}$ pp collisions, Phys.Rev.Lett. 109 (2012) 032001. 
[7] ATLAS Collaboration, (2012), Search for pair-produced heavy quarks decaying to Wq in the two-lepton channel at $\sqrt{s}=7$ TeV with the ATLAS detector, Phys. Rev. D, 86 (2012) 012007.

[8] ATLAS Collaboration, The ATLAS Experiment at the CERN Large Hadron Collider, JINST 3, S08003 (2008). 